\title{
Context-dependent Personalised Feedback Prioritisation in Exploratory Learning for Mathematical Generalisation
}

\author{
Mihaela Cocea and George Magoulas \\ London Knowledge Lab, Birkbeck College, \\ 23-29 Emerald Street, WC1N 3QS, London, UK \\ \{mihaela, gmagoulas\}@dcs.bbk.ac.uk
}

\begin{abstract}
In this paper we address the problem of prioritising feedback on the basis of multiple heterogeneous pieces of information in exploratory learning. The problem arises when multiple types of feedback are required in order to address different types of conceptual difficulties, accommodate particular learning behaviours identified during exploration, and provide appropriate support depending on the learning mode (e.g. individual or collaborative learning) and/or the stage of the exploratory learning process. We propose an approach that integrates learners' characteristics and context-related information through a Multicriteria Decision-Making formalism. The outcome is a context-aware mechanism for prioritising personalised feedback that is tested in an exploratory learning environment for mathematical generalisation.
\end{abstract}

Key words: context-dependent personalised feedback, feedback prioritisation, Analytic Hierarchy Process, Multicriteria Decision Making

\section{Introduction}

In exploratory learning, tasks can be approached in many different ways and are often characterized by some key points the learner needs to address or be aware of. The actions of learners can indicate what they need help with, but their personal characteristics may not guarantee the effectiveness of help. Context could bring valuable information that would make help more appropriate and, thus, more effective. Context-awareness has been studied in a diversity of domains like artificial intelligence [1], ubiquitous computing [2], educational psychology [3] and recommender systems [4]. The definition of context is also diverse, varying from the wide social context to the specificity of network characteristics.

In this paper we present a context-dependent personalised feedback prioritisation mechanism using the Analytic Hierarchy Process [5], a popular method in Multicriteria Decision-Making [6]. In our approach context refers to the learning mode (i.e. individual or collaborative) and to the stages within a task. The approach is illustrated using an Exploratory Learning Environment (ELE) for mathematical generalisation and the prioritisations delivered by the proposed method are validated by experts in the field of mathematical education. 
The paper is organised as follows. Section 2 briefly introduces adaptive feedback, mathematical generalisation and the system employed; Section 3 presents the multicriteria decision problem and the Analytic Hierarchy Process method. Section 4 includes examples of how this approach operates under different contextual requirements and discusses the results, and Section 5 concludes the paper.

\section{Adaptive Feedback in Exploratory Learning for Mathematical Generalisation}

Feedback is usually a response to the actions of a learner aiming to correct future iterations of the actions [7]. It includes information about what happened or did not happen as a consequence of the user's actions in relations to the goal [8]; this information is given to the users to compare their performance with the expected one [9] and to make use of it in the following attempt [8].

In exploratory learning, the freedom given to learners leads to situations when feedback is required on several aspects. This is also the case of eXpresser ${ }^{1}$ [10] [11], which is an ELE for mathematical generalisation that aims to link the visual with the algebraic-like representation of rules. It enables constructions of patterns, creating dependences between them, naming properties of patterns and creating algebraic-like rules with either names or numbers. Some screenshots are displayed in Figure 1, illustrating the system, two constructions, the properties list of a pattern that is dependent on another one, the properties list of an independent pattern and two examples of rules.

The main area of the screen in Figure 1 displays two constructions. These are solutions of two learners working independently on a task called "footpath", which is typical in the UK curriculum. The task requires to find out the number of green tiles needed to surround any pattern of red tiles (representing the footpath). The components of Construction 1 are displayed separately for ease of understanding; this construction has four patterns: (a) two compact rows of green (lighter colour) tiles and (b) two rows with gaps in between tiles: one green and one red (darker colour). The first two mentioned are the same, and consequently, have the same properties displayed in the property list of the highlighted row in Construction 1. The first property, i.e. number of iterations, shows that the pattern depends on the red one because the number of iterations of the green tiles is set to 'the number of red tiles multiplied by 2 plus 1'; the $T$ box with the name red and the corresponding value of 3 is called an icon variable and is used to make a pattern dependent on another; the use of $i$ con variables leads to general constructions, i.e. they work for any number of red tiles. The second property, moving left, is set to 1 and the third property, moving down, is set to 0 , which makes the pattern a row; for the red pattern moving left is set to 2 and moving down is set to 0 , which makes a row with gaps between the tiles. The last property establishes the number needed to colour all the tiles in the

\footnotetext{
${ }^{1}$ Developed in the context of MiGen Project, funded by the ESRC/EPSRC Teaching and Learning Research Programme (RES-139-25-0381); http://www.migen.org.
} 
pattern; in the current case it is the same as the number of iterations in the pattern. However, if a pattern is a group of several tiles, this would not be the case anymore; for example, if a pattern is a group of three tiles and is iterated five times, the number required to colour it would be three times five.

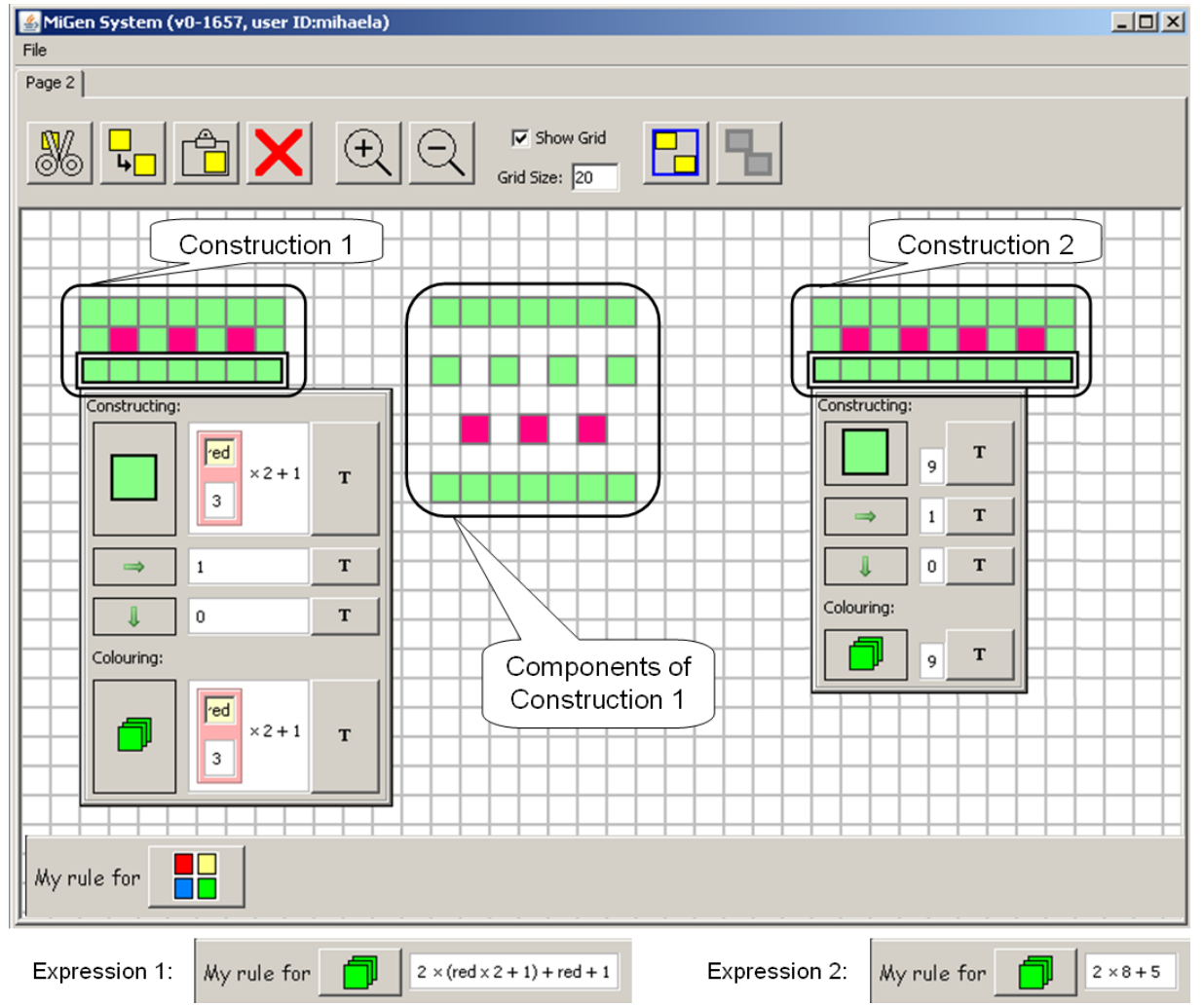

Fig. 1. eXpresser screenshots. The screenshot of the system includes a toolbar, an area for pattern construction and an area for defining rules; the toolbar (at the top) allows the following actions: cut, copy, paste, delete, zoom in, zoom out, show grid, grid size (changeable from here or using the zoom tools), group and ungroup; the main area has two constructions for the "footpath" task and two property lists; the components of Construction 1 are also presented separately. The two screenshots at the bottom illustrate the rules defined by the learners who built the two constructions.

Construction 2 is build in a similar fashion, but the compact rows of green tiles do not depend on the red pattern: the first property (number of iterations) from the property list is set to 9. At the bottom of Figure 1, two expressions corresponding to the two constructions are displayed. Expression 1 uses the name red for the number of red tiles, while Expression 2 is numeric.

In the constructions of Figure 1, both learners follow the same strategy in surrounding the footpath: two rows of tiles at top and bottom, and one row of tiles in the gaps of the red pattern; also, for both constructions, the row of green 
tiles with gaps in between (the middle one) does not depend on the red pattern and the expressions do not correspond to their corresponding constructions. However, there are a few differences: (a) they work with a different number of red tiles, i.e. 3 and 4 , respectively; (b) the first learner is very close to a general solution, while the second is still working with the particular case of 4 red tiles; (c) the expression of the first learner (Expression 1 in Figure 1) is already general, while the expression of the second learner (Expression 2 in Figure 1) is numeric.

Construction 2 could be used at this point to illustrate how the need for feedback prioritisation emerges during exploration. In this instance, from pedagogical point of view, several issues need to be addressed: (a) the construction is correct only when the red pattern consists of four tiles, i.e. it is specific, whilst the aim of the activity is to create a general construction that would work for any number of tiles; (b) the learner may need to be reminded how to make a pattern dependent on another (i.e. the use of icon variables); (c) the expression does not correspond to the construction and contains a mistake; (d) the expression is specific. To this end, different types of feedback are needed depending on learner's characteristics and contextual information. In the next section, we describe an approach that leads to prioritising feedback on these issues based on a multicriteria decision making method called the Analytic Hierarchy Process.

\section{Analytic Hierarchy Process Formalism}

Multicriteria Decision Making (MDM) defines a class of problems where a decision from a predefined set of alternatives needs to be reached by taking into account two or more criteria. Each alternative is evaluated on the set of criteria; the outcomes provide a means of comparison between the alternatives that will facilitate a selection of one or more alternatives, or a ranking between them. Other purposes are classification of alternatives into groups (clustering) and group ranking [6]. Among the possible approaches of decision problems that correspond to this description are: statistical techniques, multi-attribute utility analysis, analytic hierarchy process, knowledge bases, mathematical models, etc.

MDM has many applications in fields where decisions need to be taken. The Analytic Hierarchy Process (AHP) is one of the most popular methods in MDM and is widely applied in a diversity of areas like logistics, military, manufacturing and health-care [12]. Frequently AHP is used in combination with other methods - a recent literature review [12] reports five main categories of tools integrated with AHP: (a) mathematical programming, (b) quality function development, (c) meta-heuristics, (d) SWOT analysis, and (e) data envelopment analysis. Four works related to higher education are reported in areas of IT-based project selection [13], teaching method selection [14], education requirement selection [15] and faculty course assignment [16].

In the area of learner/user modelling, AHP has been used in combination with fuzzy logic [17] for student diagnosis in an adaptive hypermedia educational system and in combination with Multi-Attribute Utility Theory (MAUT), another 
method from MDM, in recommender systems [18], where the evaluation function from MAUT is used to rate how well each alternative fulfills the decision criteria.

The AHP uses a hierarchy to represent a decision problem and to establish priorities between alternatives depending on a set of criteria involved in the decision process. It includes three main steps: (a) construction of the hierarchy; (b) analysis of priorities and (c) verification of consistency.

The hierarchy has the general structure presented in Figure 2. The highest level represents the goal, which, in our context, is personalised feedback. The second level includes the criteria based on which the decision should be taken; in our case, the criteria refer to the learning mode and the stage in the exploratory task. The third level includes the alternatives to be prioritised with respect to the criteria; the alternatives correspond to pedagogical aspects of mathematical generalisation. The first step includes a decomposition of the decision problem into parts defined by all relevant attributes; these attributes are arranged into hierarchical levels so as to reach the hierarchical structure presented in Figure 2.

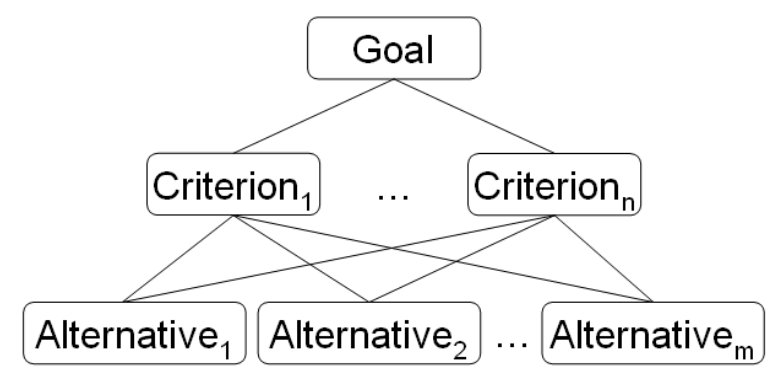

Fig. 2. Hierarchy in the Analytic Hierarchy Process

The analysis of priorities includes pairwise comparisons used to compute weights for the alternatives, which establish an order between them. This involves two steps: (a) decide priorities between criteria; (b) decide priorities between alternatives with respect to each criterion. The priorities take the form of matrices as in (1): one for the first step (priorities amongst criteria) and $n$ for the second (priorities amongst alternatives) (a matrix for each criterion). For both types of matrices the values below the main diagonal are the reversed values from above the main diagonal, i.e. $c_{j i}=1 / c_{i j}, a_{j i}=1 / a_{i j}$, as the comparison result between objects $A$ and $B$ is reversed when the order changes ( $B$ and $A$ ).

$$
C=\left[\begin{array}{cccc}
1 & c_{12} & \ldots & c_{1 n} \\
1 / c_{12} & 1 & \ldots & c_{2 n} \\
\ldots & \ldots & \ldots & \ldots \\
1 / c_{1 n} & 1 / c_{2 n} & \ldots & 1
\end{array}\right], \quad A^{L}=\left[\begin{array}{cccc}
1 & a_{12} & \ldots & a_{1 m} \\
1 / a_{12} & 1 & \ldots & a_{2 m} \\
\ldots & \ldots & \ldots & \ldots \\
1 / a_{1 m} & 1 / a_{2 m} & \ldots & 1
\end{array}\right]
$$

Each pair of criteria $c_{i}$ and $c_{j}$ has an associated value that specifies their relative importance. The values of $c_{i j}(1 \leq i, j \leq n)$ and $a_{i j}(1 \leq i, j \leq m)$ are determined using a scale from 1 to 9 , where 1 means 'equally important' and 9 means 'extremely more important'. For example, $c_{i j}=1$ means that the criteria $c_{i}$ and $c_{j}$ are equally important, $c_{i j}=3$ means that $c_{i}$ is more important than $c_{j}$ 
and $c_{i j}=9$ means that $c_{i}$ is extremely more important than $c_{j}$. The vales and meaning for the inverse pairs are: (a) $c_{j i}=1: c_{j}$ and $c_{i}$ are equally important, (b) $c_{j i}=1 / 3: c_{j}$ is less important than $c_{i}$ and (c) $c_{j i}=1 / 9: c_{j}$ is extremely less important than $c_{i}$.

The weight of each criterion is calculated using (2) and the criteria weight vector is obtained: $W=\left(w_{1}, w_{2}, \ldots, w_{n}\right)$.

$$
w_{i}=\frac{\left(\prod_{j=1}^{n} c_{i j}\right)^{1 / n}}{\sum_{i=1}^{n}\left(\prod_{j=1}^{n} c_{i j}\right)^{1 / n}}
$$

For the alternatives, a priority vector is calculated for each matrix (corresponding to a criterion) using the same equation (2). Thus priority vectors: $A\left(C r_{j}\right)=\left(A_{1}\left(C r_{j}\right), A_{2}\left(C r_{j}\right), \ldots, A_{m}\left(C r_{j}\right)\right), j=\overline{1, n}$ are obtained. Matrix A (3) results from combining the $n$ priority vectors.

$$
A=\left[\begin{array}{cccc}
A_{1}\left(C r_{1}\right) & A_{1}\left(C r_{2}\right) & \cdots & A_{1}\left(C r_{n}\right) \\
A_{2}\left(C r_{1}\right) & A_{2}\left(C r_{2}\right) & \cdots & A_{2}\left(C r_{n}\right) \\
\vdots & \vdots & \ddots & \vdots \\
A_{m}\left(C r_{1}\right) & A_{m}\left(C r_{2}\right) & \cdots & A_{m}\left(C r_{n}\right)
\end{array}\right]
$$

By combining the criteria weights and the priority vectors the final alternatives priorities vector $P$ with respect to all criteria is obtained using: $P=A * W$. More specifically, the priority for each alternative is calculated as: $p_{i}=A_{i}\left(C r_{1}\right) *$ $w_{1}+A_{i}\left(C r_{2}\right) * w_{2}+\ldots+A_{i}\left(C r_{n}\right) * w_{n}, i=\overline{1, m}$.

Consistency refers to the lack of logical contradictions in the pairwise comparisons; for example, if in a matrix the alternative $x$ is more important than alternative $y$ and less important than alternative $z$, and, at the same time, $y$ is more important than $z$, there is an inconsistency ( $x$ is more important than $z$ (by transitivity through $\mathrm{y}$ ) and $x$ is less important that $z$ by direct comparison). To verify the consistency of the $n+1$ pairwise comparisons matrices ( $n$ alternatives matrices and 1 criteria matrix), an approximation of the maximum eigenvalue for each matrix, denoted as $\lambda_{\max }$ (see Equation 4) is used to calculate the consistency index (CI). Equation (5) shows how to calculate CI for the criteria matrix and the $n$ alternatives matrices.

$$
\begin{array}{r}
\lambda_{\max _{j}}=\left(\sum_{i=1}^{m} a_{i 1}, \sum_{i=1}^{m} a_{i 2}, \ldots, \sum_{i=1}^{m} a_{i m}\right) * \\
\left(A_{1}\left(C r_{j}\right), A_{2}\left(C r_{j}\right), \ldots, A_{m}\left(C r_{j}\right)\right)^{T}, j=\overline{1, n} \\
\text { For criteria: } C I=\frac{\lambda_{\max }-n}{n-1}
\end{array}
$$

$$
\text { For alternatives: } C I_{j}=\frac{\lambda_{\text {max }_{j}}-m}{m-1}, j=\overline{1, m}
$$

$\mathrm{CI}$ and the Random Consistency Index (RCI) are used to calculate the consistency ratio (CR) as: $C R=\frac{C I}{R C I}$. The values of the RCI [5] for 1 to 10 criteria 
are displayed in Table 1 . Values of the consistency ratio below 0.10 indicate consistency, while greater values indicate the opposite. In the later case, revision of the pairwise comparisons is necessary.

Table 1. Values of RCI for $n=\overline{1,10}$.

\begin{tabular}{l|cccccccccc}
\hline $\mathrm{n}$ & 1 & 2 & 3 & 4 & 5 & 6 & 7 & 8 & 9 & 10 \\
\hline $\mathrm{RCI}$ & 0 & 0 & 0.58 & 0.90 & 1.12 & 1.24 & 1.32 & 1.41 & 1.45 & 1.49 \\
\hline
\end{tabular}

The overall consistency of the hierarchy is a function of the consistency indexes of all pairwise matrices, the RCI for the number of criteria and number of alternatives and the weights of the criteria, as in (6).

$$
C R=\frac{C I_{\text {criteria }}+w_{1} * C I_{\text {alt }_{C r_{1}}}+w_{2} * C I_{\text {alt }_{C r_{2}}}+\ldots+w_{n} * C I_{\text {alt }_{C r_{n}}}}{R C I_{n}+w_{1} * R C I_{m}+w_{2} * R C I_{m}+\ldots+w_{n} * R C I_{m}}
$$

Summarising, the AHP process involves three main steps: definition of the hierarchy, analysis of pairwise comparisons and verification of consistency. These are illustrated through scenarios in the following section.

\section{AHP for Context-Dependent Personalised Feedback Prioritisation}

Three scenarios are presented to illustrate the AHP process in the context of eXpresser and similar tasks to "footpath". The hierarchy of the AHP formalism is illustrated in Figure 3: the goal is to obtain feedback priorities; the criteria is the learning mode, i.e. individual or collaborative, and the stage in a task, i.e. specific and general. The alternatives are feedback on the following aspects: (a) correctness of construction (CC); (b) correctness of expression (CE); (c) construction-expression correspondence (C-E); (d) symmetry of construction (Sym); (e) generality of construction (CGen); (f) generality of expression (EGen); (g) use of icon variables (IV).

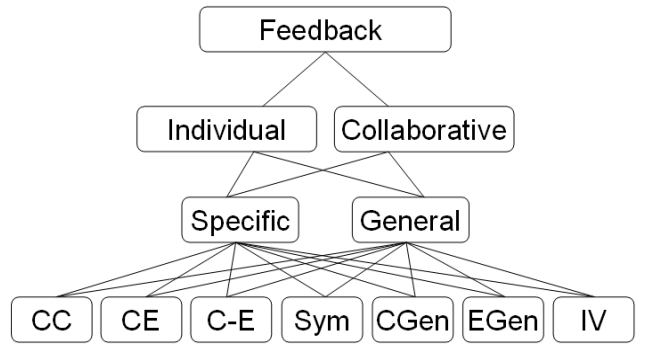

Fig. 3. AHP hierarchy.

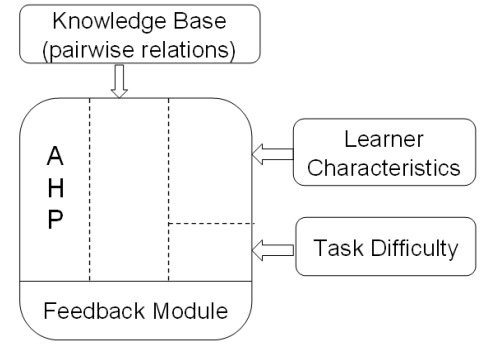

Fig. 4. Feedback module.

The pairwise comparisons between criteria and between alternatives vary depending on learner's (dynamic) characteristics: (a) level of experience (stored 
for each level of task difficulty), (b) arithmetics knowledge level and (c) preferred approach: from specific to general (S-to-G) or from general to specific (G-to$\mathrm{S})$. The feedback module (Figure 4) integrates this information together with information about task difficulty to retrieve sets of pairwise relations from the Knowledge Base. This generates different instantiations of the AHP process. To illustrate how AHP is going to operate in different situations, three scenarios are considered below (summarised in Table 2).

Table 2. Scenarios characteristics.

\begin{tabular}{lccc}
\hline Characteristics & Scenario 1 & Scenario 2 & Scenario 3 \\
\hline Mode & individual & individual & collaborative \\
\hline Task difficulty & medium & medium & medium \\
\hline Experience & low & medium & low\&medium \\
\hline Arithmetics & high & low & high\&low \\
\hline Approach & G-to-S & S-to-G & G-to-S\&S-to-G \\
\hline
\end{tabular}

The constructions for the scenarios are displayed in Figure 1: Constructions 1 and 2 are used in Scenario 1 and 2, respectively. In the collaborative scenario, i.e. Scenario 3, the learners who produced these constructions and their corresponding expressions are working together. The pairing for collaboration is made based on the similarity of the strategy [19] and the complementarity of approach and/or arithmetic level. A diagnosis of the learners' constructions [19] is carried out at the same time with the computation of feedback priorities. Combining these two sources, a decision is taken with regard to necessary and/or relevant

Scenario 1. Feedback prioritisation is established by taking into consideration: (a) the individual learning mode, (b) the learner's characteristics mentioned in Table 2 and (c) Construction 1 and Expression 1 from Figure 1. The criteria pairwise comparison, the corresponding weights and consistency information are displayed in Table 3; the alternatives pairwise comparison with respect to the criteria (specific and general context), the priority vectors and the consistency measures are displayed in Table 4 and Table 5 . The final priorities and the overall consistency are displayed in Table 6 . From these tables, the numbers assigned by the designer of the AHP component are the criteria and the alternatives pairwise comparisons; the rest are computed using the formulas presented in Section 2.

Table 3. Criteria pairwise comparison, weights, and consistency.

\begin{tabular}{l|cc|c}
\hline Criteria & Specific & General & Weights \\
\hline Specific & 1 & $1 / 2$ & 0.33 \\
General & 2 & 1 & 0.67 \\
\hline \multicolumn{4}{l}{$\lambda_{\max }=2.00, C I=0, C R=0$} \\
\hline
\end{tabular}

As the learner prefers the general-to-specific approach, the top item for feedback is icon variables as they allow general constructions. The next two items to give feedback on are correctness of construction and its generality. Construc- 
Table 4. Alternatives pairwise comparison, priority vector with respect to the specific context, and consistency.

\begin{tabular}{l|ccccccc|c}
\hline Alternatives & CC & CE & C-E & Sym & CGen & EGen & IV & Priority vector \\
\hline CC & 1 & 2 & 2 & 5 & 2 & 3 & $1 / 2$ & 0.22 \\
CE & $1 / 2$ & 1 & $1 / 2$ & 3 & $1 / 5$ & $1 / 2$ & $1 / 2$ & 0.08 \\
C-E & $1 / 2$ & 2 & 1 & 3 & $1 / 5$ & 2 & $1 / 2$ & 0.11 \\
Sym & $1 / 5$ & $1 / 3$ & $1 / 3$ & 1 & $1 / 3$ & $1 / 3$ & $1 / 3$ & 0.04 \\
CGen & $1 / 2$ & 5 & 5 & 3 & 1 & 3 & $1 / 2$ & 0.22 \\
EGen & $1 / 3$ & 2 & $1 / 2$ & 3 & $1 / 3$ & 1 & $1 / 2$ & 0.09 \\
IV & 2 & 2 & 2 & 3 & 2 & 2 & 1 & 0.23 \\
\hline$\lambda_{\max }=7.75, C I=0.13, C R=0.10$ & $C R=0$
\end{tabular}

Table 5. Alternatives pairwise comparison, priority vector with respect to the general context, and consistency.

\begin{tabular}{l|ccccccc|c}
\hline Alternatives & CC & CE & C-E & Sym & CGen & EGen & IV & Priority vector \\
\hline CC & 1 & 5 & 5 & 7 & 1 & 5 & $1 / 2$ & 0.27 \\
CE & $1 / 5$ & 1 & $1 / 2$ & 7 & $1 / 5$ & 2 & $1 / 3$ & 0.08 \\
C-E & $1 / 5$ & 2 & 1 & 3 & $1 / 3$ & 3 & $1 / 3$ & 0.10 \\
Sym & $1 / 7$ & $1 / 7$ & $1 / 3$ & 1 & $1 / 3$ & 3 & $1 / 3$ & 0.05 \\
CGen & 1 & 5 & 3 & 3 & 1 & 2 & $1 / 2$ & 0.19 \\
EGen & $1 / 5$ & $1 / 2$ & $1 / 3$ & $1 / 3$ & $1 / 2$ & 1 & $1 / 5$ & 0.04 \\
IV & 2 & 3 & 3 & 3 & 2 & 5 & 1 & 0.27 \\
\hline$\lambda_{\max }=7.82, C I=0.14, C R=0.10$ & $C R=0.10$
\end{tabular}

Table 6. Scenario 1: Feedback priorities and overall consistency.

\begin{tabular}{l|ccccccc}
\hline Alternatives & CC & CE & C-E & Sym & CGen & EGen & IV \\
\hline Priorities & 0.25 & 0.08 & 0.10 & 0.05 & 0.20 & 0.06 & 0.26 \\
\hline Overall $C R=0.04$ & & & & & & \\
\hline
\end{tabular}

tion 1 has two general components and a specific one, which indicates that the learner has used icon variables, so no feedback on that is necessary; as the construction is correct, the first feedback to be provided will be on the generality of the construction, and more specifically, on the generality of the only specific component of the construction. From the AHP process, the next priorities are related to the expression: correspondence between construction and expression, correctness of expression and expression generality. The last two items are already in place, so no feedback on them is given. If in the previous step the learner has made the specific component general, the construction would correspond to the expression; if not, feedback would be provided to the learner to make sure the construction (partially general) corresponds to the expression (general).

Scenario 2. In this scenario, the prioritisation is computed for the individual learning mode, taking in consideration the learner's characteristics displayed in Table 2, Construction 2 and its corresponding expression from Figure 1. The 
procedure is applied as in Scenario 1; only the final feedback priorities and the overall consistency are reported in Table 7 .

Table 7. Scenario 2: Feedback priorities and overall consistency.

\begin{tabular}{l|ccccccc}
\hline Alternatives & CC & CE & C-E & Sym & CGen & EGen & IV \\
\hline Priorities & 0.310 & 0.108 & 0.130 & 0.194 & 0.092 & 0.060 & 0.106 \\
\hline
\end{tabular}

As the learner prefers a specific-to-general approach, the feedback addresses generality at the end. The first aspects to give feedback on are: the correctness of construction, its symmetry and the correspondence between construction and expression. The first two aspects are in place, so the feedback would be given on the correspondence between expression and construction. If the learner addresses this aspect and the new expression is $2 * 9+5$, the feedback on the following item, i.e. correctness of construction, becomes unnecessary. If the learner does not correct the expression accordingly, the feedback would address the correctness of expression, pointing out that the construction is correct and that the expression should correspond to the construction. So, feedback at this point includes the two interrelated aspects: the correctness of expression and the correspondence between construction and expression. Only after establishing the correctness of construction and expression for the specific case of 4 red tiles, the feedback will address the generality of the construction: the use of icon variables, the generality of construction, and, finally, the generality of expression.

Scenario 3. In the collaborative mode, the two learners are working together towards finding a general solution. The first leaner has a construction with 3 red tiles, while the second has a construction with 4 red tiles. Consequently, a specific approach on one side will lead to an inadequate construction on the other, which enforces the learners to work with the general. The feedback priorities for this particular collaborative situation are displayed in Table 8.

Table 8. Scenario 3: Feedback priorities and overall consistency.

\begin{tabular}{l|ccccccc}
\hline Alternatives & CC & CE & C-E & Sym & CGen & EGen & IV \\
\hline Priorities & 0.15 & 0.08 & 0.11 & 0.24 & 0.17 & 0.05 & 0.19 \\
\hline \multicolumn{2}{l}{ Overall $C R=0.03$} & & & & & & \\
\hline
\end{tabular}

As the learners are 'forced' to work with the general, the first aspect to give feedback on is the symmetry of construction as, otherwise, it would be difficult to make it general - as both learners have symmetric constructions, this is not necessary. The next aspect to give feedback on is the use of icon variables; ideally, this feedback from the system would be replaced by the feedback of learner one to learner two, who has a specific construction. The next two aspects to be addressed are the generality and the correctness of the construction. For the same reason mentioned previously, the construction will be correct only when it is general, so generality is addressed first and correctness afterwards. The 
expression is dealt with at the end, starting from the correspondence with the construction, addressing its correctness and finally, its generality.

The priorities delivered by the AHP process were validated by two experts in the field of mathematical education who were aware of the way learners interacted with eXpresser. Both of them agreed on the prioritisation for the two individual situations, but there was one disagreement on the collaborative scenario. One expert agreed with the prioritisation delivered by the AHP process, while the other argued for the following order: IV, CGen, CC, Sym, C-E, CE and EGen. This order differs from the output of the AHP process by the fact that symmetry is moved from the first place to the fourth. The expert's argument for this was that they could build a construction that is correct and not symmetrical, but symmetry becomes important at this point because it would facilitate finding a general expression. On the other hand, the other expert argued that symmetry is important from the very beginning to facilitate the generality of construction (and then, the expression) because one of the learners prefers the specific-to-general approach and also has a low arithmetics ability; therefore, even if the other learner would be able to reach a general construction, though non-symmetrical, and to find a corresponding expression, for the other learner this would be difficult and hardly beneficial.

\section{Concluding Remarks}

In this paper we have presented a mechanism for personalised feedback prioritisation depending on the learning mode, i.e. individual or collaborative, the context within a task, i.e. specific or general, and the learner's characteristics. The way the mechanism operates was illustrated in two individual and one collaborative scenario. The feedback priorities for the individual mode were confirmed by two experts, whilst the priority given to symmetry in collaborative mode was considered by one of the experts as too high. One possible explanation for the diversity of the experts' opinion could be the added complexity of the collaborative mode, which is an issue that requires further investigation.

\section{Acknowledgements}

This work is partially funded by the ESRC/EPSRC Teaching and Learning Research Programme (Technology Enhanced Learning); Award no: RES-13925-0381).

\section{References}

1. Akman, V., Bouquet, P., Thomason, R., Young, R.A.: Modeling and Using Context: Third International and Interdisciplinary Conference, CONTEXT, 2001, Dundee, UK, July 27-30, LNAI, vol. 2116. Springer (2001)

2. Kwon, O.: The potential roles of context-aware computing technology in optimization-based intelligent decision-making. Expert Systems with Applications, 31(3), 629-642 (2006) 
3. Wang, S.S., Treat, T.A., Brownell, K.D.: Cognitive Processing About ClassroomRelevant Contexts: Teachers' Attention to and Utilization of Girls' Body Size, Ethnicity, Attractiveness, and Facial Affect. Journal of Educational Psychology, 100(2), 473-489 (2008)

4. Anand, S.S. Mobasher, B.: Contextual Recommendation. In B. Berendt, A. Hotho, D. Mladenic, G. Semeraro (Eds.), From Web to Social Web: Discovering and Deploying User and Content Profiles, LNCS, vol. 4737, pp. 142-160. Springer (2007)

5. Saaty, T.L.: The Analytic Hierarchy Process. New York: McGraw-Hill (1980).

6. Zopounidis, C., Doumpos, M.: Multicriteria classification and sorting methods: A literature review. European Journal of Operational Research, 138(2), 229-246 (2002)

7. Mason, B.J., Bruning, R.: Providing feedback in computer-based instruction: What the research tells us (2001) http://dwb.unl.edu/Edit/MB/MasonBruning.html

8. Wiggins, G.: Feedback: how learning occurs (2008) http://www . authenticeducation .org/bigideas/article. lasso?artId=61

9. Johnson, D.W., Johnson, R.T.: Cooperative learning and feedback in technologybased instruction. In Dempsey, J.V. and Sales, G.C. (Eds.): Interactive Instruction and Feedback, Educational Technology, Englewood Cliffs, NJ (1993)

10. Pearce, D., Geraniou, E. Mavrikis, M., Gutierrez-Santos, S., Kahn, K.: Using Pattern Construction and Analysis in an Exploratory Learning Environment for Understanding Mathematical Generalisation: The Potential for Intelligent Support. In S. Gutierrez-Santos, M. Mavrikis (eds.), Proceedings of the 1st International Workshop on Intelligent Support for Exploratory Environments, EC-TEL'08 (2008).

11. Noss, R. Hoyles, C. Geraniou, E. Gutierrez-Santos, S. Mavrikis, M., Pearce, D.: Broadening the sense of 'dynamic': an intelligent system to support students' mathematical generalisation. Submitted to The International Journal on Mathematics Education (2008).

12. Ho, W.: Integrated analytic hierarchy process and its applications A literature review. European Journal of Operational Research, 186, 211-228 (2008)

13. Kwak, N.K., Lee, C.W.: A multicriteria decision-making approach to university resource allocation and information infrastructure planning. European Journal of Operational Research, 110(2), 234-242 (1998)

14. Lam, K., Zhao, X.: An application of quality function deployment to improve the quality of teaching. International Journal of Quality and Reliability Management, 15(4), 389-413 1998

15. Koksal, G., Egitman, A.: Planning and design on industrial engineering education quality. Computers and Industrial Engineering, 35(3-4), 639-642 (1998)

16. Ozdemir, M.S., Gasimov, R.N.: The analytic hierarchy process and multiobjective 01 faculty course assignment. European Journal of Operational Research, 157(2), 398-408 (2004)

17. Grigoriadou, M., Kornilakis, H., Papanikolaou, K.A., and Magoulas, G.D.: Fuzzy Inference for Student Diagnosis in Adaptive Educational Systems. In Vlahavas, I.P., Spyropoulos, C.D. (eds.), Methods and Applications of Artificial Intelligence: Proceedings of the 2nd Hellenic Conference on AI, SETN2002, LNAI vol. 2308, pp. 191-202. Berlin: Springer-Verlag (2002)

18. Schmitt, C., Dengler, D., Bauer, M.: Multivariate Preference Models and Decision Making with the MAUT Machine. In Brusilovsky, P., Corbett, A., de Rosis, F. (eds.) User Modeling, LNAI vol. 2702, pp. 297-302. Springer (2003)

19. Cocea, M., Magoulas, G.: Combining Intelligent Methods for Learner Modelling in Exploratory Learning Environments. In Proceedings of the 1st International Workshop on Combinations of Intelligent Methods and Applications, in conjunction with ECAI 2008, pp. 13-18 (2008) 